\title{
Periodic Variability of the Brown Dwarf Kelu-1
}

\author{
F. J. Clarke \\ Institute of Astronomy, Madingley Road, Cambridge, CBз OHA, UK \\ C. G. Tinney \\ Anglo-Australian Observatory, PO Box 296, Epping, NSW 2121, Aus
}

S. T. Hodgkin

Institute of Astronomy, Madingley Road, Cambridge, CB3 OHA, UK

\begin{abstract}
We present results of two observing campaigns aimed at characterising variability of the L2 brown dwarf Kelu-1. The first campaign in March 2000 detected photometric variability at the $1.2 \%$ level, with a strong period of 1.8 hours. Followup spectroscopic observations with the VLT in February 2002 show no evidence of significant variability in the dust sensitive molecular lines, but do show evidence for variability in the $\mathrm{EW}(\mathrm{H} \alpha)$ with a period of 1.8 hours. Radial velocity measurements rule out a close substellar companion. Kelu-1 appears to be a single brown dwarf with a rotation period of 1.8 hours.
\end{abstract}

\section{Photometry in March 2000}

Kelu- 1 was observed on 2000 March 24-25 with the Taurus-2 instrument on the $3.9 \mathrm{~m}$ Anglo-Australian Telescope. A $410 \AA$ filter which selects the $\mathrm{TiO}$ and $\mathrm{CrH}$ molecular bandheads was used. Differential photometry clearly shows Kelu-1 is variable at a low level $(\sim 1 \%)$. Periodogram analysis of the lightcurve reveals a strong period of $1.80 \pm 0.05$ hours, with a peak-to-peak amplitude of $11.9 \pm 0.8$ mmag. The same 1.8 hour period is found in each nights data, indicating the source of variability is stable over at least $\sim 48$ hours ( $\sim 25$ rotations). Assuming the 1.8 hour period is the rotation period, we can place a lower limit on Kelu-1's radius of $R \geq 0.09 \pm 0.01 R_{\odot}$ - consistent with theoretical predictions (Chabrier et al, 2000). However, several models can explain the observed variability;

- Dust Cloud Meteorology: Comparison with models implies a "dusty" photosphere, with clear "holes" covering $<2 \%$ of the surface.

- A Close Binary: We may be observing ellipsoidal variability caused by a close companion to Kelu-1. In this case, we would observe two maxima per rotation, making the orbital period 3.6 hours. The amplitude of variability requires a brown dwarf companion rather than a planet $\left(\mathrm{M}>13 \mathrm{M}_{\mathrm{jup}}\right)$.

This work is reported in full in Clarke, Tinney and Covey (2002). 


\section{VLT spectroscopy in February 2002}

To further investigate Kelu-1's variability, we obtained time resolved spectroscopy of Kelu-1 on 2002 February 13 \& 14 UT with the FORS2 instrument on VLT UT4. Kelu-1 was observed for 2 hours each night in a sequence of 480 s exposures. With a resolution of about 1500 , five minute exposures provided the optimal balance between signal-to-noise ( $\sim 80$ per pixel) and phase resolution ( 5 $\min \simeq 1 / 20$ th rotation period). Thus we completely sampled two of the 1.8 hour periods found from the 2000 photometry. Data were reduced in the standard way, and the extracted spectra wavelength calibrated with the night sky lines.

\subsection{Molecular lines}

The complex of molecular lines around $8600 \AA$ ( $\mathrm{TiO}, \mathrm{CrH}$ and $\mathrm{FeH}$ ) is particularly interesting due to their sensitivity to temperature and dust physics (Allard et al, 2001). If Kelu-1 has a very inhomogeneous photosphere, we might expect to see these lines vary as it rotates (e.g. Kirkpatrick et al, 2001). The time series of spectra show no obvious changes in the molecular lines, and measurements of the band strengths show no variability. This is not, however, too surprising. The $1.2 \%$ variability detected in 2000 can be explained by surface inhomogeneity of $<2 \%$. Simplistic models (combining DUSTY and COND spectra (Allard et al, 2001)) predict $<1 \%$ change in the depth of the $\mathrm{TiO}$ and $\mathrm{CrH}$ bandheads for such a small covering fraction.

\subsection{H $\alpha$ equivalent width}

The time series spectra of the $\mathrm{H} \alpha$ line throughout our observations clearly indicate it is variable. We have measured the pseudo-equivalent width $(\mathrm{PEW}(\mathrm{H} \alpha))$ with the splot task in IRAF. Periodogram analysis of the $\mathrm{PEW}(\mathrm{H} \alpha)$ is consistent with the 1.8 hour period perviously found. There is no evidence for changes in the central wavelength of the $\mathrm{H} \alpha$ line.

\subsection{Limits on radial velocity changes}

We have used the strong metal lines of RbI $(7800,7948 \AA)$ and CsI $(8521 \AA)$ to look for radial velocity changes indicative of a binary system. We place an upper limit of $\mathrm{K}<20 \mathrm{~km} / \mathrm{s}$ on the timescale of 2-24 hours. The binary explanation for the photometric variability would imply a 3.6 hour binary period with a velocity amplitude of $40-60 \mathrm{~km} / \mathrm{s}$. We can therefore rule out Kelu-1 as a close substellar binary at the $2-3 \sigma$ level.

\section{References}

Allard, F., Hauschildt, P. H., Alexander, D. R., Tamanai, A. \& Schweitzer, A. 2001, ApJ, 556, 357

Chabrier, G., Baraffe, I., Allard, F., \& Hauschildt, P. 2000, ApJ, 542, 464

Clarke, F.J., Tinney, C.G., \& Covey, K.R. 2002, MNRAS, 332, 361

Kirkpatrick, J. D., Dahn, C. C., Monet, D. G., Reid, I. N., Gizis, J. E., Liebert, J., Burgasser, A. J. 2001, AJ, 121, 3235 